\section{Feeding Your Feelings: Emotion Regulation Strategies and Emotional Eating}

Personality and Social Psychology Bulletin 36(6) 792-804

(C) 2010 by the Society for Personality and Social Psychology, Inc Reprints and permission: sagepub.com/journalsPermissions.nav DOI: |0.1 I77/0|4616721037|383 http://pspb.sagepub.com

@SAGE

\author{
Catharine Evers', F. Marijn Stok', and Denise T. D. de Ridder'
}

\begin{abstract}
The process by which emotions affect eating behavior emerges as one of the central unresolved questions in the field of emotional eating. The present studies address the hypothesis that the regulation strategies people use to deal with these emotions are responsible for increased eating. Negative emotions were induced and intake of comfort food and non-comfort food was measured by means of taste tests. Emotion induction was preceded by measuring individual differences in emotion regulation strategies (Study I) or by instructions to regulate emotions in either an adaptive (reappraisal) or maladaptive (suppression) manner (Study 2). Study 3 also entailed a control condition without any regulation instructions. Relative to reappraisal and spontaneous expression, suppression led to increased food intake, but only of the comfort foods. Emotions themselves were not responsible for this effect. These findings provide new evidence that the way in which emotions are regulated affects eating behavior.
\end{abstract}

\title{
Keywords
}

emotional eating, emotion regulation, reappraisal, suppression

Received March 26, 2009; revision accepted November 13, 2009

Negative emotions are part and parcel of life. It has been found that in some people negative emotions lead to increased eating, a phenomenon called emotional eating (Bruch, 1973). The exact process by which emotions affect eating behavior emerges as one of the central unresolved questions in the field of emotional eating (Leith \& Baumeister, 1996). It has been suggested that it may not necessarily be the emotion itself that elicits the change in eating behavior but rather the manner in which the emotion is dealt with (Wiser \& Telch, 1999). The current studies address this hypothesis and specifically investigate whether different ways of regulating one's negative affect result in changes in eating behavior.

\section{Emotional Eating}

Negative emotions bring about a bodily state similar to satiety (Schachter, Goldman, \& Gordon, 1968), as increased autonomic emotional activity leads to the release of appetite-inhibiting hormones such as catecholamine and to a variety of gastric changes similar to those that are involved in satiety (Blair, Wing, \& Wald, 1991). The tendency to overeat in response to negative emotions, then, is surprising from a biological point of view (Wing, Blair, Epstein, \& McDermott, 1990). Also, from a functional perspective, emotional eating seems maladaptive. Emotions prepare the organism for a set of diverse actions required to respond optimally to the environmental demands (e.g., Ekman, 1992; Levenson, 1994), and eating interferes with the demands that emotions impose on individuals.

Despite being irrational, the occurrence of emotional eating has been observed among various populations. Convincing evidence supports the link between emotions and eating in eating disordered people (Polivy \& Herman, 2002; Thayer, 2001). For example, obese individuals often show increased food intake after having experienced negative emotions (Telch \& Agras, 1996). In addition, "restrained" eaterschronic dieters - seem particularly vulnerable to eat in reaction to negative emotional events (e.g., Greeno \& Wing, 1994; Polivy, Herman, \& McFarlane, 1994). However, also in healthy, nonrestrained individuals the phenomenon of emotional eating

\footnotetext{
'Utrecht University, Utrecht, Netherlands

Corresponding Author:

Catharine Evers, Department of Clinical \& Health Psychology,

Utrecht University, PO Box 80 I40, 3508 TC, Utrecht, Netherlands

Email: C.Evers@uu.nl
} 
has been observed (e.g., Newman, O’Connor, \& Conner, 2007; Nguyen-Rodriguez, Chou, Unger, \& Spruijt-Metz, 2008).

To explain the biologically and functionally irrational tendency to overeat in emotional situations, several speculations regarding the psychological mechanism behind this phenomenon have been put forward. One theory (Heatherton \& Baumeister, 1991) is that bingeing occurs as part of an attempt to escape from negative self-awareness. Attention is focused on the immediate stimulus environment and away from more meaningful levels of cognition, allowing the individual to avoid dealing with ego-threatening information. Such narrowing of attention, however, also results in disinhibition, creating a situation where individuals are likely to engage in binge episodes. Another frequently posited mechanism is that overeating increases the experience of positive emotions. Individuals derive pleasure from the consumption of food because of its qualities (taste, odor; e.g., Lehman \& Rodin, 1989) or because of the joy of eating "banned" substances (Fairburn \& Cooper, 1982). A third model, masking theory, posits that overeating is an attempt to misattribute perceived stress to eating, so as to distract from the original source of distress (Herman \& Polivy, 1988).

All these theories share the assumption that before overeating occurs, individuals experience negative affect that they cannot properly regulate, prompting them to employ a strategy they do have access to but that seems highly maladaptive: overeating (also see Wiser \& Telch, 1999). This line of reasoning has important implications because it suggests that the problem is not necessarily associated with the experience of negative emotions per se but rather with the lack of adaptive emotion regulation strategies available to regulate negative affect.

The idea that the emotion itself may not be responsible for overeating could explain why the tendency to overeat after the experience of negative emotions has not consistently been found among nonclinical individuals. As outlined above, several studies found that restrained eaters show increased eating during the experience of negative emotions (Heatherton, Herman, \& Polivy, 1991). However, a number of other studies did not replicate these findings (e.g., Chua, Touyz, \& Hill, 2004; Lowe \& Maycock, 1988), indicating that restraint status is not a necessary guarantee for emotional eating (Spoor, Bekker, Van Strien, \& Van Heck, 2007; Van Strien, 1999; Williams et al., 2002).

Among nonrestrained eaters results are also diverse, and emotions have been found to result not only in increased eating (e.g., Lowe \& Maycock, 1988) but also in decreased eating (e.g., Kenardy, Butler, Carter, \& Moor, 2003) or in unaffected eating patterns (Rotenberg \& Flood, 1999; Ruderman, 1985). A review of experimental studies investigating eating after experiencing negative emotions (Macht, 2008) indicated that among normal, nonrestrained individuals in more than $40 \%$ of the studies, participants ate more after experiencing negative affect, whereas in almost $40 \%$ of the studies, declined food intake was found. In the remaining proportion, no significant change in eating behavior was observed. As posited by the author, "It is difficult to predict how normal eaters change eating in response to emotions" (Macht, 2008, p. 3).

Striking is the finding that not even self-reported emotional eating status is a prerequisite for emotional eating. That is, individuals reporting that they have the tendency to overeat in response to diverse negative emotions, as assessed by emotional eater scales (e.g., Van Strien, Frijters, \& Bergers, 1986), have not consistently been found to increase food intake during emotional episodes. For example, some studies revealed that stressed self-reported emotional eaters indeed ate more than unstressed and nonemotional eaters ( $\mathrm{O}^{\prime} \mathrm{Connor}$, Jones, Conner, McMillan, \& Ferguson, 2008; Oliver, Wardle, \& Gibson, 2000). Other studies, however, did not find this relationship (Adriaanse, de Ridder, \& Evers, 2010; Conner, Fitter, \& Fletcher, 1999; Evers, de Ridder, \& Adriaanse, 2009). These findings also demonstrate the lacking consistency in empirical results relating to emotional eating. The present study's perspective that not so much the emotion itself but the manner in which the emotion is regulated is decisive in determining eating behavior may help to account for this bulk of inconsistent empirical findings.

\section{Emotion Regulation}

The concept of emotion regulation refers to the efforts people undertake to influence the experience and expression of their emotions (Gross, 1999). It has been demonstrated that emotion regulation is paramount to both our physical and mental health (Gross, 2007). Emotion regulation strategies can be divided into antecedent- and response-focused strategies. Antecedent-focused strategies take place before emotion response tendencies have become fully activated and have changed behavioral and peripheral physiological responding; response-focused strategies occur once an emotion has already been fully triggered, thus after response tendencies have been activated (Gross \& John, 2003). Two prototypical strategies that are commonly used in daily life, and that have been the topic of much research, are cognitive reappraisal and expressive suppression (e.g., Gross \& John, 2004; Richards $\&$ Gross, 2000). These strategies are the focus of the current studies.

Reappraisal is an antecedent strategy: It entails changing the way one thinks about an emotional situation so as to change its emotional impact once the situation occurs. It is considered a "cooling" strategy in that it provides psychological distance from the aversive situation (Mischel \& Ayduk, 2004). Expressive suppression, on the other hand, is a response strategy: It entails the reduced or nonexpression of emotions when in an emotional state (Gross \& John, 2003).

These strategies have differential consequences. Generally, reappraisal is related to less negative emotion experience 
and expression, less physiological activation, and more positive emotion experience. In contrast, suppression is associated with increases in physiological responding and decreases in behavioral expression, but it fails to decrease emotion experience (Gross, 2002). In the long term, frequent emotional suppression has been empirically linked to attachment avoidance, less social support, more symptoms of depression, lower self-esteem, less life satisfaction, and lower overall well-being (Gross \& John, 2003, 2004).

Such findings indicate that, generally, reappraisal is an adaptive emotion regulation strategy, paired with positive outcomes, whereas suppression is a maladaptive emotion regulation strategy with negative outcomes. In the present studies, we assume that the typical consequences of these emotion regulation strategies also apply to eating behavior, such that maladaptive regulation strategies are dysfunctional and result in increased emotional eating as compared to adaptive emotion regulation strategies. If the positive outcomes that generally follow adaptive emotion regulation strategies also apply for food intake, adaptive emotion regulation strategies should protect against emotional eating.

Evidence for this assumption comes from research showing that college students' scores on the Difficulties in Emotion Regulation Scale (Gratz \& Roemer, 2004) uniquely predicted the number of binge eating episodes (Whiteside et al., 2007). Evidence for adaptive emotion regulation strategies not leading to increased eating comes from Mischel's work with the delay of gratification paradigm (e.g., Metcalfe \& Mischel, 1999; Mischel \& Moore, 1973), showing that instructing children to think about food treats in a more abstract way - abstraction being a form of cognitive change similar to reappraisal-decreased their impulse to eat the treats (allowing them to obtain a favorite but delayed reward). Likewise, individuals who regularly use reappraisal as an emotion regulation strategy may show unaffected or decreased eating when experiencing emotions in comparison to individuals using maladaptive regulation strategies.

\section{The Present Studies}

The present studies investigate the effect of emotion regulation strategies on eating behavior. We hypothesize that the way in which negative emotions are regulated affect food intake rather than negative emotions per se. From a theoretical viewpoint, this hypothesis contributes over and above existing research. Beside the possibility of providing an explanation for the many discrepancies in the literature on how eating is changed in response to emotions, current psychological models regarding emotional eating (e.g., Fairburn \& Cooper, 1982; Heatherton \& Baumeister, 1991; Herman \& Polivy, 1988) typically see the act of overeating in response to negative emotions as a maladaptive emotion regulation strategy. The current hypothesis, however, allows for the possibility that overeating results from maladaptive emotion regulation.
Moreover, this also implies that the emotional experience per se is not necessarily associated with overeating.

More specifically, we hypothesized that maladaptive regulation strategies such as suppression result in increased food intake compared to adaptive regulation strategies such as reappraisal. These hypotheses are tested among healthy individuals in three studies with experimental settings. The first study investigates individual differences in the use of suppression and reappraisal and analyzes the consequences on food intake after emotions have been induced. The second study contrasts these regulation strategies by experimental manipulation. To assess whether reappraisal results in increased eating, suppression in decreased eating, or both, Study 3 also incorporates a control condition without any regulation instructions.

Emotions are induced by using recent emotional autobiographical life events (Study 1) or emotional film excerpts (Studies 2 and 3). To assess food intake, bogus taste tests were used. These tests assess actual consumption of different foods, thereby omitting the bias of self-reports or retrospective memories of eating behavior (e.g., Stice, Fisher, \& Lowe, 2004). In Study 1, the taste test contained only "comfort foods" (caloric and highly palatable food); in Studies 2 and 3, non-comfort foods were also included. The choice for different emotion inductions and providing different food types across the studies was implemented to portray the robustness of the assumed effect.

Finally, as all studies assess actual food intake, it was considered important to control for individual differences in eating styles, as tapped by the Dutch Eating Behavior Questionnaire (DEBQ; Van Strien et al., 1986). This questionnaire consists of three subscales: Emotional Eating (the desire to eat in response to several distinct emotions; e.g., "Do you have the desire to eat when you feel sad?"), External Eating (the desire to eat in response to external cues like sight or smell of food; e.g., "Do you have the desire to eat when you pass a cafeteria?"), and Restrained Eating (dieting-related behavior; e.g., "How often do you try not to eat in between meals because you are dieting?"). Answers are rated in terms of frequency using 5-point scales ranging from never to very often. As people's eating styles may interact with the food intake during the taste tests, these measures were incorporated in the analyses as control items.

\section{Data Treatment}

The weight of food consumed was calculated based on the difference in the weight of the bowl before and after the taste test. Because the different food types varied in size and weight, each food type was standardized, and $z$ scores were summed per participant to create an index of food intake. For the ease of interpretation, means are reported in grams. Because the taste tests in Studies 2 and 3 entailed comfort and non-comfort food items, factor analyses combined with 
reliability analyses were conducted to identify clusters of comfort and non-comfort foods.

\section{Study I: Individual Differences in Suppression Versus Reappraisal}

Individual differences in suppression and cognitive reappraisal were assessed. In the emotion condition, sadness was induced; in the control condition, a neutral state was induced. We predicted that when emotions are induced, individuals regularly using suppression to regulate emotions will show increased eating behavior when being emotional in comparison to individuals rarely using this strategy or individuals regularly using reappraisal.

\section{Method}

Participants. A total of 40 female university students participated in this study. Data from participants who were obese $(\mathrm{BMI}>30 ; n=3)$ were excluded from the analyses. The final sample consisted of 37 participants (average age $=$ 22.92 years) with a mean BMI of $23.13(S D=2.89)$.

Procedure. The study was presented in two supposedly unrelated parts, one about affect and one about taste. To create standardized satiety states, participants were informed that they were not allowed to eat 2 hours before participation. After signing informed consents, participants completed demographic questions and the Emotion Regulation Questionnaire (ERQ; Gross \& John, 2003), assessing individual differences in the habitual use of two emotion regulation strategies: expressive suppression and cognitive reappraisal. These two subscales are orthogonal $(r=.005)$, and the Suppression subscale contains items such as "I control my emotions by not expressing them" and "I keep my emotions to myself"; the Cognitive Reappraisal subscale contains items such as "When I'm faced with a stressful situation, I make myself think about it in a way that helps me stay calm" and "When I want to feel less negative emotion (such as sadness or anger), I change what I'm thinking about."

Next, to induce emotions, a "recall" procedure (Neumann $\&$ Waldstein, 2001) was used. In the emotion condition $(n=$ 19), participants were instructed in Step 1 to recall and reexperience a recent and personally relevant sad event. It was stressed that it had to be an event that still evoked sadness when it was brought to mind. Participants were motivated to verbalize freely, like they were talking to a friend. They were instructed to talk until they reexperienced the event again. In Step 2 participants were instructed to think in silence for 2 more min about the event. In the control condition $(n=18)$, the instructions were the same, but instead of an emotional event participants had to recall a regular, daily event.

Before and after the emotion induction, emotion experiences including sadness were assessed by asking participants how strongly they experienced 21 different emotions based on 7-point Likert-type scales ranging from 0 (not at all) to 6 (very much).

In the supposed "second study," food intake was assessed by means of bogus taste tests. Participants were provided with three bowls containing different savory and sweet foods. During the taste tests participants were provided with questionnaires, ostensibly to evaluate taste, structure, and perception of the foods, thereby ensuring that they ate at least something of every food type provided. Unbeknownst to the participants, each bowl was weighed in advance. Participants were given $10 \mathrm{~min}$ for the test (also see Lowe \& Maycock, 1988), providing them with ample time to taste. During the taste test, the experimenter left the room.

After the tasting, participants completed the DEBQ (Van Strien et al., 1986) measuring individual differences in eating behavior. These measures are considered possible influences on people's eating habits and were therefore included as control items.

Funneled debriefing procedures (Bargh \& Chartrand, 2000) were then used to assess the extent to which participants were aware of the true nature of the "unrelated studies." After participants had been debriefed and dismissed, the bowls with food were weighed again. Participants received money or course credit for participation.

\section{Materials}

Emotion experience. Because the emotion induction was focused on sadness recall, sadness ratings were the target emotions; the remaining emotion ratings were used for exploratory analyses.

Food intake. For the assessment of food intake, participants rated three types of comfort foods: chocolate, crisps, and cookies.

Emotion regulation. Individual differences in emotion regulation styles suppression ( 4 items; $\alpha=.71$ ) and reappraisal (6 items; $\alpha=.80$ ) were measured with the ERQ.

Control variables. Emotional eating (13 items; $\alpha=.89$ ), external eating (10 items; $\alpha=.78)$, and restrained eating (10 items; $\alpha=.92$ ) were assessed with the DEBQ.

\section{Results}

Manipulation check. Sadness ratings were subjected to a 2 (time: before vs. after emotion induction) $\times 2$ (condition: emotion vs. control) multivariate analysis of variance (MANOVA) with the last factor between subjects. The significant time effect, $F(1,35)=71.32, p<.001, \eta^{2}=.67$, was qualified by a significant interaction effect, $F(1,35)=71.32$, $p<.001, \eta^{2}=.67$. Simple effects revealed that the sadness ratings did not differ between conditions before the emotion induction $\left(M_{\text {emotion }}=0.68, S D=1.20\right.$ vs. $M_{\text {control }}=0.44, S D=$ $0.86 ; p>.492)$. After the induction, in the emotion condition $(M=4.16, S D=1.30)$ more sadness was reported than in the 
control condition $(M=0.44, S D=1.04), F(1,35)=91.10$, $p<.001$, Cohen's $d=3.16$. To explore if other emotions also increased because of the induction, the remaining emotion ratings were subjected to the same analysis. Beside sadness, only anger was induced. After the induction, in the emotion condition $(M=2.42, S D=1.84)$ more anger was reported than in the control condition $(M=0.56, S D=1.15), F(1,35)=$ $13.55, p<.001, d=1.21$. The anger ratings did not differ between conditions before the emotion induction $\left(M_{\text {emotion }}=\right.$ $0.37, S D=1.01$ vs. $\left.M_{\text {control }}=0.50, S D=1.15 ; p>.71\right)$. As in the emotion condition, the increase in sadness $(M=3.47)$ was significantly higher than the increase in anger $(M=2.05)$, $t(18)=4.03, p<.001, d=0.82$, the emotion induction was considered as successful.

Food intake. An analysis of variance (ANOVA) with condition as the independent variable and total food consumption as the dependent variable was performed. No significant condition effect was found $(F<1)$ : Participants in the emotion condition $(M=42.17, S D=15.67)$ consumed an amount of food equal to participants in the control condition $(M=$ $42.0, S D=17.41)$. Moreover, a regression analysis for food intake, with change in sadness and anger from before to after the recall as independent variables, revealed that both sadness $(p=.502)$ and anger $(p=.514)$ did not predict the amount of food consumed. Thus, self-reported emotions per se did not affect subsequent eating behavior. To consider individual differences in food intake, the three DEBQ subscales (External, Restrained, and Emotional Eating) were entered as covariates in the ANOVA described above. None of the covariates reached significance ( $p \mathrm{~s}>.28)$, and the main effect of condition remained nonsignificant $(F<1)$.

Effect of emotions and emotion regulation strategies on food intake. Hierarchical regression analyses were performed to test our prediction that emotion regulation strategies predict increased food intake. Variables were mean centered to minimize multicollinearity (Aiken \& West, 1991). Food intake was regressed onto the control variables age and BMI (Step 1); condition $(0=$ control and $1=$ emotion $)$, suppression, and reappraisal (Step 2); and the interaction between condition and suppression versus reappraisal (Step 3). Neither the first two steps $(p s>.30)$ nor any of the individual beta weights were significant $(p s>.17)$. At Step 2 , the variables explained $15 \%$ of the variance. Adding the interaction terms at Step 3 increased the amount of variance explained by $15 \%$, a significant increment, $F(2,29)=3.73, p=.036$. Only the beta weight associated with the interaction between suppression and condition was significant $(\beta=.664, p=.012)$, whereas none of the other betas were significant ( $p \mathrm{~s}>.13$ ). To ensure that the interaction effect was not driven by any outliers, Cook's distance $(D)$ was calculated, with $D>1$ generally regarded as an indication for outliers. $D$ ranged from 0.00 to $0.72(M=0.49, S D=0.13)$, indicating no substantial outlier problems. To further examine the nature of this interaction, simple slopes were computed for the regression of food intake

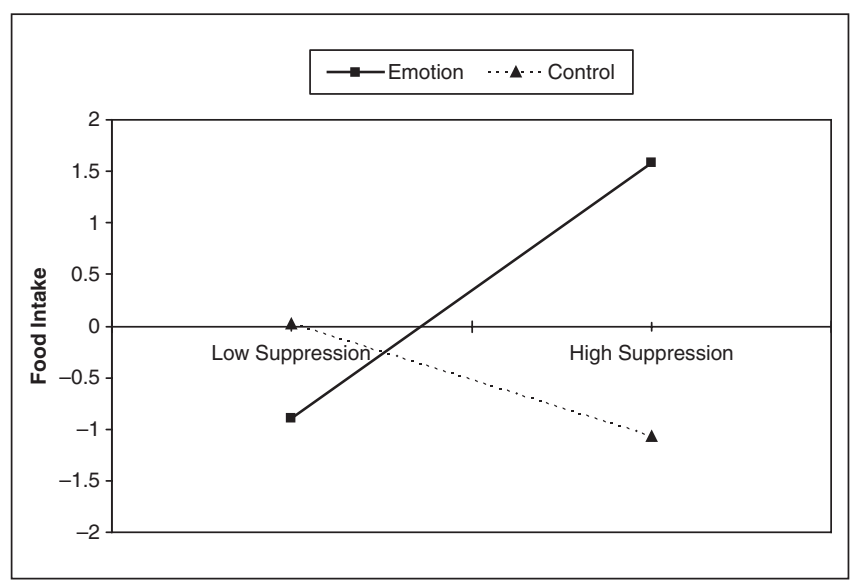

Figure I. Food intake as a function of condition (control vs. emotion) and suppression

Note: Food intake is reflected in standardized scores. Positive scores indicate more intake.

on suppression (one standard deviation below vs. above the mean suppression score; Aiken \& West, 1991; see Figure 1) for the control and emotion conditions separately. A significant slope was observed for the emotion condition $(\beta=.564$, $p=.012)$ but not for the control condition $(\beta=-.258, p=$ .297). These results indicate that a higher score on suppression was associated with increased food intake, but only in the emotion condition.

\section{Discussion}

Results of Study 1 show that individual differences in emotion regulation strategies were associated with increased food intake: Individuals regularly using suppression in their daily lives consumed more food when being emotional than individuals rarely using this strategy. Individual differences in cognitive reappraisal, however, did not relate to food intake. We assume that this unexpected finding originates from our emotion induction procedure: Cognitive reappraisal is an antecedent strategy that exerts its influence before the emotional response is fully blown. In this study, though, participants were instructed to fully reexperience sadness first. This makes it unlikely that cognitive reappraisal could still take place effectively, even among individuals who regularly use this strategy. In line with this assumption, previous research has shown that initiating reappraisal late during an emotion induction procedure indeed comes at the cost of the advantages that early reappraisal initiation typically brings forth (Sheppes \& Meiran, 2008).

\section{Study 2: Suppression and Reappraisal: An Experimental Approach}

To address the above-mentioned limitation and extend these findings, in Study 2 suppression and reappraisal were 
manipulated. Negative emotion was induced by using a film excerpt. Beside comfort food, non-comfort food was also included in the taste test. We hypothesized that participants in the suppression condition would eat more than participants in the reappraisal condition. Moreover, these differences were assumed to be especially pronounced for comfort food because these are the food types commonly expected to alleviate one's affect (Lebel, Lu, \& Dubé, 2008).

\section{Method}

Participants. In all, 50 female university students participated. Data from 3 participants were excluded because of missing values from technical failures, and data from 3 others who reported to have an eating disorder were excluded. The final sample consisted of 44 participants (average age $=$ 21.7 years, with a mean BMI of 21.64; $S D=2.18$ ).

Procedure. Aside from the emotion induction, regulation instructions, and food types, Study 2 followed the procedure of Study 1 exactly. Upon arrival at the laboratory, participants were seated behind a computer. A film excerpt was used to induce emotions, and instructions were first given on how to watch this excerpt. The instructions were based on previously established procedures (see Gross, 1998; Richards \& Gross, 2000). In the suppression condition $(n=22)$ participants were instructed not to show their feelings while watching, so that anybody looking at them would not be able to determine what kind of excerpt they were watching. The experimenter stressed that it was important to control their face and body language, making it appear as if they did not experience any emotions. In the reappraisal condition $(n=$ 22) participants were instructed to take a distant and objective perspective while watching and were told to remember that what they saw was not real, that they were just watching actors perform, and that they had to pay attention to technical aspects of the excerpt, such as the employed camera angles. In both conditions participants were told that they would be videotaped while watching, so that their reactions could be coded. This was not actually the case and was told only to encourage participants to optimally regulate their emotions according to the instructions. Participants were then shown a 4-min excerpt from the film American History $X$. The excerpt depicted a brutal and violent scene, meant to induce negative affect (Smeets, Candel, \& Merckelbach, 2004). Subsequently, the taste test was introduced.

\section{Materials}

Manipulation checks. To check whether the excerpt evoked significant increases in emotion experience, emotional experiences (see Study 1) were assessed before and after the film excerpt. Negative emotions (fear, frustration, irritation, disgust, and anger) that were validated to be increased by this excerpt in previous research (Schaefer, Nils, Sanchez, \& Philippot, 2006;
Smeets et al., 2004) were combined into a negative emotion compound (before vs. after the excerpt; $\alpha=.75$ vs. $\alpha=.78$ ). To determine whether the regulation instructions were successful, participants answered four questions on 7-point Likerttype scales (adapted from Richards \& Gross, 2000). Two questions were combined into a "suppression check" compound $(r=.299, p=.048)$ and assessed the extent to which participants, while watching the excerpt, (a) thought their facial expressions revealed their feelings and (b) were able to suppress their emotions. The other two questions were combined into a "reappraisal check" compound $(r=.633, p=.001)$ and assessed the extent to which participants, while watching the excerpt, (a) took a distant perspective and (b) paid attention to technical aspects.

Food intake. Participants tasted chocolate and crisps (intended as comfort food) and salted and unsalted crackers (intended as non-comfort food). To verify these clusters, a principal axis analysis was computed. Both the eigenvalues criterion and a visual scan of the scree plot indeed pointed to a twofactor solution. Chocolate and crisps loaded on the first factor and unsalted crackers on the second factor, as expected. The salted crackers, however, also loaded on the first factor. Thus, based on caloric value salted crackers were not considered as comfort food, but they were seemingly treated as such by the participants. An oblique (oblimin) rotation showed that the factors were not correlated $(r=.13)$, indicating that the items formed two reliably different components. To verify the factor structure, the items were further evaluated in an analysis with varimax rotation, and the same two factors were found. Reliability analyses were then conducted to form internally coherent scales. Chocolate, crisps, and salty crackers were combined into a compound reflecting comfort food $(\alpha=.80)$; the unsalted crackers were retained as a single non-comfort food item.

Control variables. Emotional $(\alpha=.90)$, external $(\alpha=.69)$, and restrained $(\alpha=.94)$ eating was assessed with the DEBQ.

\section{Results}

Manipulation checks: Emotion experience. Negative emotion experience was subjected to a 2 (condition: suppression vs. reappraisal) $\times 2$ (time: before vs. after the excerpt) ANOVA with time as the within-subjects variable. The time effect was significant, $F(1,42)=55.60, p<.001, d=1.26$, indicating that negative emotions increased significantly after watching the excerpt $\left(M_{\text {before excerpt }}=1.30, S D=0.94\right.$; to $M_{\text {after }}=2.80$, $S D=1.40)$. The nonsignificant interaction effect $(F<1)$ indicated that the two conditions did not differ in terms of inducing negative emotions (suppression condition: $M_{\text {before }}=1.22$, $\left.S D=0.84 ; M_{\text {after }}=2.86, S D=1.08\right)$ and reappraisal condition $\left(M_{\text {before }}=1.39, S D=1.05 ; M_{\text {after }}=2.75, S D=1.22\right)$. Thus, the excerpt was successful in inducing negative emotions.

Manipulation checks: Regulation instruction. The suppression and reappraisal checks were subjected to a MANOVA with 
Table I. Food Intake in Grams per Condition (Study 2)

\begin{tabular}{lrrrrr}
\hline & \multicolumn{4}{c}{ Condition } \\
\cline { 2 - 3 } & \multicolumn{2}{c}{ Suppression } & & \multicolumn{2}{c}{ Reappraisal } \\
\cline { 2 - 3 } \cline { 5 - 6 } Food type & $M$ & $S D$ & & $M$ & $S D$ \\
\hline Comfort food & 44.63 & 32.44 & & 21.82 & $17.70^{*}$ \\
Non-comfort food & 13.50 & 7.84 & & 13.05 & 16.00 \\
\hline * & & & & &
\end{tabular}

condition as independent variable. The multivariate condition effect was significant, $F(2,42)=14.21, p<.001, d=0.49$. As expected, univariate analyses indicated that participants in the suppression condition employed the suppression strategy more $(M=4.13, S D=1.08)$ than participants in the reappraisal condition $(M=2.59, S D=0.96), F(1,43)=25.51$, $p<.001, d=1.51$, whereas participants instructed to reappraise employed the reappraisal strategy more $(M=3.25$, $S D=0.75)$ than participants instructed to suppress $(M=$ 2.46, $S D=1.21), F(1,43)=6.87, p=.012, d=0.78$.

Food intake. The intake of comfort and non-comfort food was subjected to a MANOVA with condition as independent variable. The multivariate effect was significant, $F(2,41)=$ $2.87, p=.027, d=0.75$. Univariate analyses showed that participants in the suppression condition consumed more comfort foods than participants in the reappraisal condition, $F(1,42)=7.99, p=.007, d=0.88$ (see Table 1 for the means). The effect for non-comfort food was not significant $(F<1)$, indicating that participants consumed equal amounts of noncomfort food in both conditions. To assess if emotion experience influenced food intake, two regression analyses were conducted, one for comfort food and another for non-comfort food, with change in negative emotions from before to after the excerpt as the independent variable. The negative emotion compound predicted neither the amount of comfort food consumed $(p=.414)$, nor the amount of non-comfort food consumed $(p=.093)$. Self-reported emotion experience per se, therefore, did not affect subsequent eating behavior.

Control variables. To control for individual differences in eating behavior, the DEBQ scales (emotional, external, and restrained eating) were entered as covariates in the MANOVA described above. None of the covariates reached significance ( $p \mathrm{~s}>.125)$, whereas the main effect of condition remained significant, $F(2,37)=3.42, p=.043$.

\section{Discussion}

Confirming the hypotheses, findings revealed that participants who were instructed to suppress the expression of their emotions ate more comfort foods than participants who were instructed to reappraise these emotions. There was no effect of emotions per se on food intake. Although results reveal that suppression is responsible for increased food intake during negative emotional encounters as compared to reappraisal, it remains unclear whether maladaptive regulation strategies result in increased eating or adaptive emotion regulation strategies in decreased eating, or both, as compared to a neutral control condition. Study 3 aimed to address this limitation and to replicate the findings of Study 2.

\section{Study 3: Suppression, Reappraisal, and Spontaneous Expression}

The procedure of Study 2 was replicated with two adjustments. A control condition was added, involving a "no regulation" condition, so that each regulation strategy could be compared to anything participants do when they are not instructed to regulate (based on Gross, 1998). Importantly, such control condition provides a rather conservative comparison with the regulation conditions as participants are actually free to regulate if they choose to (Gross, 2002). Moreover, because Study 2 entailed only one comfort food type, salted crackers were replaced by unsalted crackers. We hypothesized that participants in the suppression condition would eat more comfort foods than participants in the reappraisal condition. The inclusion of the control condition served to explore whether suppression promotes or reappraisal dampens increased eating, or both.

\section{Method}

Participants. A total of 63 female university students participated. Data from one participant were excluded because she consumed more than 3 standard deviations above average of all food types. The final sample consisted of 62 participants (average age $=21.5$ years) with a mean BMI of $22.46(S D=2.85)$.

Procedure. Study 3 followed the procedure of Study 2 exactly. Beside a suppression $(n=21)$ and reappraisal $(n=$ $20)$ condition, a control condition $(n=21)$ was added. In this latter condition participants were told to watch the film excerpt, without any further instructions.

\section{Materials}

Manipulation checks. The same items as in Study 2 were combined into a negative emotion compound (before vs. after the excerpt; $\alpha=.71$ vs. $\alpha=.85$ ). To determine whether the regulation instructions were successful, the same items as in Study 2 were combined into a "suppression check" $(r=.416$, $p<.001)$ and a "reappraisal check" $(r=.457, p<.001)$. As the control condition involved a "no regulation" condition where participants were free in how to react to the film, the "control check" entailed one item addressing how spontaneous participants reacted to the film excerpt ("To what extent did you express your emotions spontaneously while watching the excerpt?": adapted from Richards \& Gross, 2000). 
Food intake. Participants tasted chocolate and crisps (high in caloric value and palatability) and unsalted crackers and wholegrain biscuits (low in caloric value and palatability). As factor analyses verified this two-factor solution, chocolate and crisps were combined into a compound reflecting comfort food; the unsalted crackers and wholegrain biscuits reflected non-comfort food.

Control variables. Emotional $(\alpha=.89)$, external $(\alpha=.68)$, and restrained $(\alpha=.91)$ eating were assessed with the DEBQ.

\section{Results}

Manipulation checks: Emotion experience. Negative emotion experience was subjected to a 3 (condition: suppression vs. reappraisal vs. control) $\times 2$ (time: before vs. after the excerpt) ANOVA with time as the within-subjects variable. The time effect was significant, $F(1,59)=125.26, p<.001$, indicating that negative emotions increased significantly after watching the excerpt ( $M_{\text {before excerpt }}=0.56, S D=0.62 ; M_{\text {after }}=2.37$, $S D=1.38 ; d=1.69)$. The nonsignificant interaction effect $(p>.118)$ indicated that the three conditions did not differ in terms of inducing negative emotions (suppression condition: $M_{\text {before }}=0.55, S D=0.68 ; M_{\text {after }}=2.63, S D=1.36$; reappraisal condition: $M_{\text {before }}=0.68, S D=0.74 ; M_{\text {after }}=2.00, S D=1.38$; control condition: $M_{\text {before }}=0.47, S D=0.42 ; M_{\text {after }}=2.45$, $S D=1.38$ ). Thus, replicating Study 2, the manipulation was successful in inducing negative feelings.

Manipulation checks: Regulation instruction. The regulation checks were subjected to a MANOVA with condition as the independent variable. The multivariate condition effect was significant, $F(6,116)=6.48, p<.001$, and this effect was significant for all checks: $F \mathrm{~s}(2,59)>12.34, p \mathrm{~s}<.029$. To further analyze these effects, $t$ tests were performed. The suppression check indicated that participants in the suppression condition employed the suppression strategy more $(M=$ $4.00, S D=1.14)$ than participants in the reappraisal $(M=3.33$, $S D=1.13)$ and control conditions $(M=2.02, S D=0.80), t(39)=$ $1.91, p=.046, d=0.59$ and $t(40)=6.51, p<.001, d=2.01$, respectively. The difference between the reappraisal and control conditions was also significant, $t(39)=4.28, p<.001$, $d=1.33$. The reappraisal check showed that participants in the reappraisal condition employed the reappraisal strategy slightly more $(M=3.68, S D=0.86)$ than participants in the suppression condition $(M=2.95, S D=1.41), t(39)=1.96$, $p=.057, d=0.63$, and more than participants in the control condition $(M=2.60, S D=1.45), t(39)=2.87, p=.007, d=$ 0.91 . The suppression and control conditions did not differ on the reappraisal check $(p=.424)$. The control check revealed that participants in the control condition scored higher on the control check $(M=3.67, S D=1.35)$ than participants in the suppression condition $(M=2.33, S D=1.56)$ and the reappraisal condition $(M=2.50, S D=1.47), t(40)=2.88, p=.006$, $d=0.92$ and $t(39)=2.65, p=.046, d=0.83$, respectively.
Table 2. Food Intake in Grams per Condition (Study 3)

\begin{tabular}{|c|c|c|c|c|c|c|}
\hline \multirow[b]{3}{*}{ Food type } & \multicolumn{6}{|c|}{ Condition } \\
\hline & \multicolumn{2}{|c|}{ Suppression } & \multicolumn{2}{|c|}{ Reappraisal } & \multicolumn{2}{|c|}{ Control } \\
\hline & M & $S D$ & $M$ & $S D$ & $M$ & $S D$ \\
\hline Comfort food & 37.62 & $20.8 I_{a}$ & 25.75 & $15.56_{b}$ & 21.95 & $15.52_{b}$ \\
\hline Non-comfort food & 19.00 & $13.08_{a}$ & 15.50 & $5.53_{a}$ & $12.7 \mid$ & $4.17_{\mathrm{a}}$ \\
\hline
\end{tabular}

Note: Means in the same row that do not share subscripts differ at $p<.04$.

The suppression and reappraisal conditions did not differ on this check $(p=.449)$.

Food intake. The intake of comfort and non-comfort food was subjected to a MANOVA with condition as the independent variable. The multivariate effect was significant, $F(4$, $118)=2.56, p=.042$. Univariate analyses showed that this effect was significant for comfort food, $F(2,59)=4.44, p=$ .016 , but not for non-comfort food ( $p>.16$ ). Follow-up $t$ tests revealed that participants consumed significantly more comfort food in the suppression condition compared to both the reappraisal, $t(39)=2.01, p=.05, d=0.63$, and control conditions, $t(40)=2.76, p=.01, d=0.85$ (see Table 2 for the means). The reappraisal and control conditions did not differ significantly $(p=.39)$. To assess if emotion experience influenced food intake, two regression analyses were conducted, one for comfort food and another for non-comfort food, with change in negative emotions from before to after the excerpt as the independent variable. Negative emotions did not predict any of the consumed food types (comfort food $p=.818$, non-comfort food $p=.728$ ). Thus, self-reported emotion experience again did not affect subsequent eating behavior.

Control variables. To control for individual differences in food intake, the DEBQ scales (emotional, external, and restrained eating) were entered as covariates in the MANOVA described above. The covariates emotional and external eating were not significant ( $p \mathrm{~s}>.526$ ); external eating was a significant covariate, $F(2,55)=3.80, p=.028$. This significance, however, did not affect any of the effects as described above. That is, the multivariate condition effect for food intake remained significant, $F(4,112)=2.94, p=.024$, and at univariate level the effect for comfort food also remained significant, $F(2,56)=5.42, p=.007$.

\section{Discussion}

Findings revealed that participants who were instructed to suppress the expression of their negative emotions ate more comfort foods compared to participants who were instructed to reappraise these emotions (in line with Study 2) and compared to participants who were allowed to express their emotions spontaneously. The reappraisal and control condition 
did not differ in food consumption. These results indicate that applying maladaptive emotion regulation strategies is responsible for increased eating. Again, emotions per se did not affect food intake.

\section{General Discussion}

In the present studies, the link between emotion regulation and emotional eating was investigated. Rather than focusing on emotional experience as being responsible for increased food intake, we assumed that different strategies that individuals employ to regulate these emotions are responsible for changes in eating behavior. Adaptive and maladaptive emotion regulation strategies were compared across three experiments among female participants with normal weight and without eating pathology. Rather than self-reported food intake, actual food consumption was measured, and both individual difference and experimental approaches were applied. It was found that maladaptive emotion regulation strategies resulted in increased food intake in comparison to adaptive strategies and in comparison to spontaneous emotion expression. This was the case, however, only for comfort food types.

\section{Different Consequences of Emotion Regulation Strategies}

Suppression can be regarded as a maladaptive emotion regulation strategy, reappraisal as an adaptive one (Gross \& John, 2003; John \& Gross, 2004). Although the consequences of these strategies have been intensively investigated, the area of eating behavior was not incorporated before. The present studies addressed this untouched area, and results for suppression are in accordance with previous findings: As higher consumption of calorie-rich snack foods can certainly be considered a negative outcome healthwise, suppression resulted in unhealthier behavior than did reappraisal and than did spontaneous expression.

These consequences of the different emotion regulation strategies were not from decreases in negative emotions on the part of reappraisers, increases on the part of suppressors, or changes in general on the part of those who spontaneously expressed their emotions. The intensity of emotion experience as such did not affect food intake. These findings were replicated across two distinct emotional encounters. The finding that differential ways of coping with negative emotions are responsible for changes in eating behavior raises the question of how emotion regulation affects food intake.

According to resource depletion theory, all acts of selfcontrol rely on the same limited resource (e.g., Baumeister, Bratslavsky, Muraven, \& Tice, 1998). As such, the use of these resources for an initial act of self-control would reduce the resources available for a subsequent self-control task. It has been shown that suppression is depleting: Participants performed poorly at different self-control tasks after emotion suppression (e.g., Baumeister et al., 1998). A compelling example is Vohs and Heatherton's (2000) finding that dieters increased ice cream consumption after having suppressed their emotions, whereas this was not the case when they were allowed to express their emotions freely. They reasoned that dieters consumed more after emotion suppression because their self-regulatory resource was depleted, causing them to fail to control their eating behavior properly. Importantly, this effect was not present for nondieters, which was attributed to nondieters not requiring to exercise control over their eating behavior (and thus not experiencing the effects of lacking self-control resources). However, in our study all participants typically suppressing their emotions in daily life consumed more when being emotional (Study 1) and all participants in the suppress conditions consumed more (Studies 2 and 3), regardless of dieting status. Importantly, the mean score for restrained eating was average according to the norms, with a wide variety ranging from very low to very high. Moreover, our research investigated different forms of emotion regulation, whereas studies within the resource depletion line did not investigate if different forms of emotion regulation are different in depleting self-control resources.

Studies explicitly comparing the cognitive costs of manipulated suppression versus reappraisal revealed, in line with resource depletion theory, that reappraisal led to better memory recall than suppression (Dillon, Ritchey, Johnson, \& Labar, 2007; Richards \& Gross, 2000). Sheppes and associates, however, illustrated in a recent series of studies that not all forms of reappraisal come free of charge (Sheppes \& Meiran, 2007, 2008). The crucial aspect regarding the extent to which emotion regulation strategies are effortful seems to be the timing of the effort. When reappraisal is applied at a point in time when the emotional response has not completely evolved yet, it poses minimal challenges to self-control; when it is applied after this "optimal" time point, however, it requires effort just like suppression does (Sheppes \& Meiran, 2008).

All together, we are unable to separate which of the many differences between reappraisal and suppression was responsible for the observed effects in food intake: The current results do not allow us to conclude if (and if so, to what extent) resource depletion is responsible for the current findings. To address these questions, future research is necessary in which other forms of emotion regulation, such as thought suppression (Wegner, 1994), rumination (Nolen-Hoeksema, 1998), and distraction (Sheppes \& Meiran, 2008), are considered. A complementary approach would be to manipulate emotion regulation strategies in such a way that each strategy is equally depleting and then investigate their effects on food intake.

Alternatively, the changed food intake after emotion regulation may also find its origin in a different theoretical model than the limited resource model. For example, according to 
the hot-cool system framework (Metcalfe \& Mischel, 1999; Mischel \& Moore, 1973) there are two interacting selfregulatory systems, a "cool" cognitive system and a "hot" emotional system. It is assumed that individuals in hot states are impulsive and focused on instant gratification. In cool states, however, individuals are thinking, planning, focused on controlling the self, and delaying gratification. It has been demonstrated that reappraisal is a typical cooling strategy, whereas suppression is a heating up approach (Mischel \& Ayduk, 2004). From the distinction described above, it seems reasonable that people in the hot suppressing condition are less able to exert control over their eating habits than people in the reappraisal or control conditions and were more focused on instant gratification.

As no differences in food intake emerged between participants in the reappraisal and no-regulation conditions, the present findings did not point toward the cool reappraising strategy serving as a buffer function against emotional eating. At this point we can only speculate why this was the case. For example, spontaneous expression may have been equally functional to reappraisal for this specific emotional event, but this does not necessarily generalize to other emotional encounters. For example, previous research has revealed that emotional eaters are particularly vulnerable to increased eating in response to ego-threatening stressors (Wallis \& Hetherington, 2004). In such encounters where participants themselves are the actor, a cooling strategy such as reappraisal may exert more influence with the consequence that it may indeed protect against emotional eating. More research is obviously needed to further investigate these issues. In general, our findings reveal that emotion regulation strategies affect intake of comfort food; however, how these strategies precisely affect changes in eating behavior remains to be assessed.

\section{Implications}

The findings presented here address an important gap in the literature regarding the underlying psychological mechanism of emotional eating by showing that emotion regulation strategies shape differences in eating behavior, instead of emotions themselves. Our results thus serve as an important link in understanding how different approaches to emotional events may have profound consequences for health. For example, changing how one thinks about life events could change subsequent health behavior.

Furthermore, current models explaining emotional eating generally assume that emotional eating in itself is a maladaptive emotion regulation strategy. Thus, when negative affect is experienced, people regulate this affect by overeating, which in itself is highly maladaptive (Wiser \& Telch, 1999). Our findings, however, add to the literature by revealing that the time spectrum at which the problem occurs may already occur earlier: That is, when negative affect is experienced and people regulate this affect in a maladaptive way, this maladaptive emotion regulation strategy can be responsible for increased eating.

In addition, within the eating literature the focus has typically been on individual differences in eating behavior, like restrained eating. However, because emotional eating also manifests itself in healthy and nonrestrained samples (e.g., Nguyen-Rodriguez et al., 2008), the present results indicate that it may be prudent to also include individual difference measures tapping the affective realm.

Furthermore, these findings have important implications for the conceptualization of emotional eating, as they suggest that it may be driven by the way in which we regulate our emotions. A better conceptualization of emotional eating is especially important because it is deemed by some to be one of the central factors underlying obesity (Thayer, 2001). As obesity is one of the major present-day epidemics in the United States (Holden, 2004), a better understanding of emotional eating is of vital importance.

Moreover, these findings may also have implications for the way we try to counter emotional eating, as more effective preventive measures might be discovered. The finding that emotional eating is driven by the manner of emotion regulation is actually a promising result: Although it may sometimes be impossible to avoid experiencing negative emotions, it may be possible (though perhaps complicated) to change the way we regulate these emotions and thereby remove an important instigator of emotional eating.

\section{Limitations and Future Directions}

Although the present studies represent a promising first step regarding emotion regulation strategies as an explaining mechanism behind emotional eating, we acknowledge some limitations. First, although the present studies revealed that maladaptive emotion regulation is responsible for increased food intake during negative emotional encounters, it remains to be assessed which process is exactly responsible for these findings.

Related to this, it would be important to assess the affective consequences of emotional eating: If people lack adaptive emotion regulation strategies to regulate negative affect and consequently consume comfort foods to deal with their affect, the consumption of these foods should indeed alleviate the negative affect if emotional eating serves a regulation function. To date, hardly any studies have examined whether eating actually decreases the impact of the emotional encounter that evoked the negative emotional experience, an exception being a study by Tice, Bratslavsky, and Baumeister (2001). They induced a negative mood, and consequently participants joined in a taste test. Eating did not have any effects on mood. Some studies indirectly investigating this subject suggest 
that eating when being emotional may even increase new negative feelings such as guilt (Dubé, Lebel, \& Lu, 2005; Macht \& Dettmer, 2006).

Moreover, our studies were limited to using relatively brief emotion-induction episodes. Exposure to encounters that evoke longer lasting emotional experiences (e.g., ego-threatening emotions) may be more impactful, and under such conditions emotion regulation strategies regarded as adaptive may serve a buffer function against emotional eating.

Finally, the present studies entailed only female participants to create homogeneous groups and because emotional eating is considered to be a primarily female phenomenon (Grunberg \& Straub, 1992; Van Strien et al., 1986). Whether the current findings generalize to a male population should be addressed in future research.

Despite these limitations, the current studies provide new insight into the area of emotional eating. They suggest that the concept of emotional eating may not refer simply to eating when feeling negative but rather to eating when negative emotions are regulated in unhealthy ways. Although more research is certainly needed, this modified conception of emotional eating opens promising new alleys toward a better understanding of emotional eating.

\section{Declaration of Conflicts of Interest}

The authors had no conflicts of interest with respect to the authorship or the publication of this article.

\section{Funding}

The authors received no financial support for the research and/or authorship of this article.

\section{References}

Adriaanse, M., de Ridder, D. T. D., \& Evers, C. (2010). Emotional eating: Eating when emotional or emotional about eating? Psychology \& Health. DOI: 10.1080/08870440903207627, 1-17.

Aiken, L. S., \& West, S. G. (1991). Multiple regression: Testing and interpreting interactions. Thousand Oaks, CA: Sage.

Bargh, J. A., \& Chartrand, T. L. (2000). The mind in the middle: A practical guide to priming and automaticity research. In H. T. Reis \& C. M. Judd (Eds.), Handbook of research methods in social and personality psychology (pp. 253-285). New York, NY: Cambridge University Press.

Baumeister, R. F., Bratslavsky, E., Muraven, M., \& Tice, D. M. (1998). Self-control depletion: Is the active self a limited resource? Journal of Personality and Social Psychology, 74, 1252-1265.

Blair, E. H., Wing, R. R., \& Wald, A. (1991). The effects of laboratory stressors on glycemic control and gastrointestinal transit time. Psychosomatic Medicine, 53, 133-143.

Bruch, H. (1973). Eating disorders: Obesity, anorexia nervosa, and the person within. New York, NY: Basic Books.

Chua, J. L., Touyz, S., \& Hill, A. J. (2004). Negative mood-induced overeating in obese binge eating: An experimental study. International Journal of Obesity, 28, 606-610.
Conner, M., Fitter, M., \& Fletcher, W. (1999). Stress and snacking: A diary study of daily hassles and between-meal snacking. Psychology \& Health, 14, 51-63.

Dillon, D. G., Ritchey, M., Johnson, B. D., \& Labar, K. S. (2007). Dissociable effects of conscious emotion regulation strategies on explicit and implicit memory. Emotion, 7, 354-365.

Dubé, L., Lebel, J. L., \& Lu, J. (2005). Affect asymmetry and comfort food consumption. Physiology \& Behavior, 86, 559-567.

Ekman, P. (1992). An argument for basic emotions. Cognition and Emotion, 6, 169-200.

Evers, C., de Ridder, D. T. D., \& Adriaanse, M. A. (2009). Assessing yourself as an emotional eater: Mission impossible? Health Psychology, 28, 717-725.

Fairburn, C. G., \& Cooper, P. J. (1982). Self-induced vomiting and bulimia nervosa: An undetected problem. British Medical Journal, 284, 1153-1155.

Gratz, K. L., \& Roemer, L. (2004). Multidimensional assessment of emotion regulation and dysregulation: Development, factor structure, and initial validation of the Difficulties in Emotion Regulation Scale. Journal of Psychopathology and Behavioral Assessment, 26, 41-54.

Greeno, C. G., \& Wing, R. R. (1994). Stress-induced eating. Psychological Bulletin, 115, 444-464.

Gross, J. J. (1998). Antecedent- and response-focused emotion regulation: Divergent consequences for experience, expression, and physiology. Journal of Personality and Social Psychology, 74, 224-237.

Gross, J. J. (1999). Emotion regulation: Past, present, future. Cognition and Emotion, 13, 551-573.

Gross, J. J. (2002). Emotion regulation: Affective, cognitive, and social consequences. Psychophysiology, 39, 281-291.

Gross, J. J. (2007). Handbook of emotion regulation. New York, NY: Guilford.

Gross, J. J., \& John, O. P. (2003). Individual differences in two emotion regulation processes: Implications for affect, relationships, and well-being. Journal of Personality and Social Psychology, $85,348-362$.

Gross, J. J., \& John, O. P. (2004). Healthy and unhealthy emotion regulation: Personality processes, individual differences, and life span development. Journal of Personality, 72, 1301-1333.

Grunberg, N. E., \& Straub, R. O. (1992). The role of gender and taste class in the effects of stress on eating. Health Psychology, $11,97-100$.

Heatherton, T. F., \& Baumeister, R. F. (1991). Binge eating as escape from self-awareness. Psychological Bulletin, 110, 86-108.

Heatherton, T. F., Herman, C. P., \& Polivy, J. (1991). Effects of physical threat and ego threat on eating behavior. Journal of Personality and Social Psychology, 60, 138-143.

Herman, C. P., \& Polivy, J. (1988). Excess and restraint in bulimia. In K. Pirke, W. Vandereycken, \& E. Ploog (Eds.), The psychobiology of bulimia (pp. 33-41). New York, NY: SpringerVerlag.

Holden, C. (2004). Obesity watch. Science, 305, 1236. 
John, O. P., \& Gross, J. J. (2004). Healthy and unhealthy emotion regulation: Personality processes, individual differences, and lifespan development. Journal of Personality, 72, 1301-1334.

Kenardy, J., Butler, A., Carter, C., \& Moor, S. (2003). Eating, mood, and gender in a noneating disorder population. Eating Behaviors, 4, 149-158.

Lebel, J. L., Lu, J., \& Dubé, L. (2008). Weakened biological signals: Highly-developed eating schemas among women are associated with maladaptive patterns of comfort food consumption. Physiology and Behavior, 94, 384-392.

Lehman, A. K., \& Rodin, J. (1989). Styles of self-nurturance and disordered eating. Journal of Consulting and Clinical Psychology, 57, 117-122.

Leith, K. P., \& Baumeister, R. F. (1996). Why do bad moods increase self-deflating behaviour? Emotion, risk taking, and self-regulation. Journal of Personality and Social Psychology, $71,1250-1267$.

Levenson, R. W. (1994). Human emotions: A functional view. In P. Ekman \& R. J. Davidson (Eds.), The nature of emotion: Fundamental questions (pp. 123-126). New York, NY: John Wiley.

Lowe, M. R., \& Maycock, B. (1988). Restraint, disinhibition, hunger and negative affect eating. Addictive Behaviors, 13, 369-377.

Macht, M. (2008). How emotions affect eating: A five-way model. Appetite, 50, 1-11.

Macht, M., \& Dettmer, D. (2006). Everyday mood and emotions after eating a chocolate bar or an apple. Appetite, 46, 332-336.

Metcalfe, J., \& Mischel, W. (1999). A hot/cool-system analysis of delay of gratification: Dynamics of willpower. Psychological Review, 106, 3-19.

Mischel, W., \& Ayduk, O. (2004). Willpower in a cognitiveaffective processing system: The dynamics of delay of gratification. In R. F. Baumeister \& K. D. Vohs (Eds.), Handbook of self-regulation: Research, theory and practice (pp. 99-129). New York, NY: Guilford.

Mischel, W., \& Moore, B. (1973). Effects of attention to symbolically presented rewards on self-control. Journal of Personality and Social Psychology, 28, 172-179.

Neumann, S. A., \& Waldstein, S. R. (2001). Similar patterns of cardiovascular response during emotional activation as a function of affective valence and arousal and gender. Journal of Psychosomatic Research, 50, 245-253.

Newman, E., O'Connor, D. B., \& Conner, M. (2007). Daily hassles and eating behaviour: The role of cortisol reactivity status. Psychoneuroendocrinology, 32, 125-132.

Nguyen-Rodriguez, S. T., Chou, C., Unger, J. B., \& Spruijt-Metz, D. (2008). BMI as a moderator of perceived stress and emotional eating in adolescents. Eating Behaviors, 9, 238-246.

Nolen-Hoeksema, S. (1998). The other end of the continuum: The costs of rumination. Psychological Inquiry, 9, 216-219.

O'Connor, D. B., Jones, F., Conner, M., McMillan, B., \& Ferguson, E. (2008). Effects of daily hassles and eating style on eating behavior. Health Psychology, 27, s20-s31.
Oliver, G., Wardle, J., \& Gibson, E. L. (2000). Stress and food choice: A laboratory study. Psychosomatic Medicine, 62, 853-865.

Polivy, J., \& Herman, C. P. (2002). Causes of eating disorders. Annual Review of Psychology, 53, 187-213.

Polivy, J., Herman, C. P., \& McFarlane, T. (1994). Effects of anxiety on eating: Does palatability moderate distress-induced overeating in dieters? Journal of Abnormal Psychology, 103, 505-510.

Richards, J. M., \& Gross, J. J. (2000). Emotion regulation and memory: The cognitive costs of keeping one's cool. Journal of Personality and Social Psychology, 79, 410-424.

Rotenberg, K. J., \& Flood, D. (1999). Loneliness, dysphoria, dietary restraint, and eating behavior. International Journal of Eating Disorders, 25, 55-64.

Ruderman, A. J. (1985). Dysphoric mood and overeating: A test of restraint theory's disinhibition hypothesis. Journal of Abnormal Psychology, 94, 78-85.

Schachter, S., Goldman, R., \& Gordon, A. (1968). Effect of fear, food deprivation, and obesity on eating. Journal of Personality and Social Psychology, 10, 91-97.

Schaefer, A., Nils, F., Sanchez, X., \& Philippot, P. (2006). A multi-criteria validation of 70 emotion-inducing films (Tech. rep.). Louvain-La-Neuve, Belgium: Université Catholique de Louvain.

Sheppes, G., \& Meiran, N. (2007). Better late than never? On the dynamics of online regulation of sadness using distraction and cognitive reappraisal. Personality and Social Psychology Bulletin, 33, 1518-1532.

Sheppes, G., \& Meiran, N. (2008). Divergent cognitive costs for online forms of reappraisal and distraction. Emotion, 8 , 870-874.

Smeets, T., Candel, I., \& Merckelbach, H. (2004). Accuracy, completeness, and consistency of emotional memories. American Journal of Psychology, 117, 595-610.

Spoor, S. T. P., Bekker, M. H. J., Van Strien, T., \& Van Heck, G. L. (2007). Relations between negative affect, coping, and emotional eating. Appetite, 48, 368-376.

Stice, E., Fisher, M., \& Lowe, M. R. (2004). Are dietary restraint scales valid measures of acute dietary restriction? Unobtrusive observational data suggest not. Psychological Assessment, 16, 51-59.

Telch, C. F., \& Agras, W. S. (1996). Do emotional states influence binge-eating in the obese? International Journal of Eating Disorders, 20, 271-279.

Thayer, R. E. (2001). Calm energy. How people regulate mood with food and exercise. New York, NY: Oxford University Press.

Tice, D. M., Bratslavsky, E., \& Baumeister, R. F. (2001). Emotional distress regulation takes precedence over impulse control: If you feel bad, do it! Journal of Personality and Social Psychology, 80, 53-67.

Van Strien, T. (1999). Success and failure in the measurement of restraint: Notes and data. International Journal of Eating Disorders, $28,460-464$. 
Van Strien, T., Frijters, J. E. R., \& Bergers, G. P. A. (1986). The Dutch Eating Behavior Questionnaire (DEBQ) for assessment of restrained, emotional, and external eating behavior. International Journal of Eating Disorders, 5, 295-315.

Vohs, K. D., \& Heatherton, T. F. (2000). Self-regulatory failure: A resource-depletion approach. Psychological Science, 11, 249-254.

Wallis, D. J., \& Hetherington, M. M. (2004). Stress and eating: The effects of ego-threat and cognitive demand on food intake in restrained and emotional eaters. Appetite, 43, 39-46.

Wegner, D. M. (1994). Ironic processes of mental control. Psychological Review, 101, 34-52.

Whiteside, U., Chen, E., Neighbors, C., Hunter, D., Lo, T., \& Larimer, M. (2007). Difficulties regulation emotions: Do binge eaters have fewer strategies to modulate and tolerate negative affect? Eating Behaviors, 8, 162-169.

Williams, J. M. G., Healy, H., Eade, J., Windle, G., Cowen, P. J., Green, M. W., et al. (2002). Mood, eating behaviour and attention. Psychological Medicine, 32, 469-481.

Wing, R. R., Blair, E. H., Epstein, L. H., \& McDermott, M. D. (1990). Psychological stress and glucose metabolism in obese and normal-weight subjects: A possible mechanism for differences in stress-induced eating. Health Psychology, 9, 639-700.

Wiser, S., \& Telch, C. F. (1999). Dialectical behavior therapy for binge eating disorder. Journal of Clinical Psychology, 55, 755-768. 